\title{
A successful lifestyle intervention model replicated in diverse clinical settings
}

\author{
S Mark, ${ }^{1}$ MSc, PhD; S du Toit, ${ }^{2}$ MD; T D Noakes, ${ }^{3}$ MD, DSc; K Nordli; ${ }^{2}$ D Coetzee, ${ }^{4}$ MD; M Makin, ${ }^{4}$ MD; $\mathbf{S}$ van der Spuy, ${ }^{4}$ MD; \\ J Frey, ${ }^{4} \mathrm{MD} ; \mathrm{J}$ Wortman, ${ }^{5} \mathrm{MD}$ \\ ${ }^{1}$ Approach Analytics, Nanaimo, British Columbia, Canada \\ ${ }^{2}$ Valemount Health Center, British Columbia, Canada \\ ${ }^{3}$ Department of Human Biology, Faculty of Health Sciences, University of Cape Town; Sports Science Institute of South Africa, Cape Town, South Africa \\ ${ }^{4}$ Omineca Medical Clinic, Vanderhoof, British Columbia, Canada \\ ${ }^{5}$ Department of Family Practice, Faculty of Medicine, University of British Columbia, Vancouver, Canada
}

Corresponding author: S Mark (sean@approachanalytics.com)

Lifestyle interventions (LIs) can treat metabolic syndrome and prevent type 2 diabetes mellitus, but they remain underutilised in routine practice. In 2010, an LI model was created in a rural primary care practice and spread with few resources to four other rural practices. A retrospective chart review evaluated changes in health indicators in two practice environments by following 372 participants, mainly women (mean age 52 years). Participants had a mean body mass index of $37 \mathrm{~kg} / \mathrm{m}^{2}$ at baseline and lost an average of $12 \%$ of their initial body weight as a result of the intervention. Among participants at the first intervention site for whom cardiometabolic data were available, the prevalence of metabolic syndrome decreased from $58 \%$ at baseline to $19 \%$ at follow-up. Taken as a whole, our experience suggests that LIs are feasible and deliver meaningful results in routine primary care practice.

S Afr Med J 2016;106(8):763-766. DOI:10.7196/SAMJ.2016.v106i8.10136

Lifestyle interventions (LIs) can treat metabolic syndrome and reduce the incidence of type 2 diabetes mellitus (T2DM) in high-risk individuals. ${ }^{[1]}$ However, realising the health benefits of LIs in routine clinical practice remains elusive. ${ }^{[2]}$ In January 2010, an LI model was created in a rural primary care practice and spread to four other rural communities. We present changes in health indicators among participants in two physician-led interventions.

\section{Methods}

Ethics approval was obtained from the Northern Health Authority and the University of British Columbia (Ref. no. H10-02573), Canada. The intervention was open to both individuals wanting to lose weight and those interested in a non-pharmacological approach to managing insulin resistance. The foundation of the intervention model was group medical visits, with 15 - 25 participants overseen by a clinical facilitator.

The clinically facilitated meetings featured a presentation germane to living a healthier lifestyle, such as sugar addiction, medication management, maintaining adherence while on vacation, etc. The remainder of the meeting time was used to answer participants' questions and address experiences relating to living a healthier lifestyle. At the first site (S1), programme length was determined by participants' progress towards their health goals. At the second site (S2), the intervention was 3 months in length. Individuals requiring additional support were encouraged to form peer-led support groups on their own initiative.

A quality improvement process whereby various lifestyle prescriptions were tested with different groups was used to refine the intervention at S1. Results from these non-randomised 'trials' were tracked using local electronic medical record data. The outcome of this process was an intervention featuring a two-stage diet programme. The weight-loss diet restricted calories to approximately 1100 and $1500 \mathrm{kcal} /$ day for women and men, respectively; participants were instructed to avoid foods containing sugar and other refined carbohydrates, in addition to restricting the consumption of dietary fat. To assist in appetite control, participants were instructed not to undertake moderate or vigorous physical activity until they had reached their weight-loss goal. After reaching their target weight, a high-fat diet was used for weight maintenance. The use of a high-fat diet was predicated on the high prevalence of insulin resistance in the patient population and favourable changes in multiple health indicators in randomised trials of up to 2 years' duration in such populations. ${ }^{[3]}$ Foods consumed on the maintenance diet included beef, poultry, fish, eggs, oils, moderate amounts of hard cheeses, and small amounts of nuts, nut butters, seeds and berries.

\section{Measurements}

Height was measured using a stadiometer, with participants wearing no shoes. Waist circumference was measured with a flexible tape measure and with the help of another participant. Weight was measured at every group visit with participants wearing light indoor clothing. Participants completed the PHQ-9 questionnaires to assess their mood. Scores on the PHQ-9 range from 0 (absence of depressive symptoms) to 27 (severe depressive symptoms). A score of $\geq 10$ on the PHQ-9 was used to indicate depression.

\section{Evaluation rationale, data extraction and statistical analyses}

Following the success of the intervention at S1, the intervention model spread to four other rural communities. The intervention was created in 2010 by a physician (SDT) working in a service contract environment and was adopted into the practices of four fee-for-service physicians, a nurse practitioner and two registered nurses. The initial evaluation was planned to document health changes at $\mathrm{S} 1$, but owing to the unanticipated spread of the intervention, weight-loss results were included in the evaluation from four fee-for-service physicians. 
The clinical and community observations from the other sites, which have not been formally evaluated, were consistent with the health improvements reported here.

Data were extracted from an electronic medical system during the most active periods of the interventions; this was early 2010 to 2011 for S1, while for S2 anthropometric data were retrieved beginning in early 2012 . For S1, where cardiometabolic data were available, baseline data were sought no earlier than January 2008. The time between the first and last weighing was used to define the time spent in the LI by participants at S1, as participants were weighed at each meeting. Intensity of participation in the LI was calculated by dividing the months in the programme by the number of visits. We used International Diabetes Federation crite$\mathrm{ria}^{[4]}$ to classify participants as having metabolic syndrome (MetS). A haemoglobin Alc (HbAlc) measurement was used to indicate the presence of diabetes, in accordance with local clinical protocols.

Differences between anthropometric and biochemical variables were calculated, and paired $t$-tests were used to assess whether baseline values were statistically different to those at follow-up. Owing to the small sample size of individuals with $\mathrm{HbAlc}$ measurements ( $n=18$ ), the Wilcoxon signedrank test was used to test whether followup values were significantly different from baseline. We used multivariable analysis to examine the predictors of changes in body mass index (BMI) between baseline and follow-up in participants at S1. All statistical analyses were conducted on SAS version 9.2 (SAS Institute, USA).

\section{Resullts}

This study documented the creation and replication of a successful LI in rural British Columbia (BC). We evaluated health improvements among 372 participants at two physician-led interventions in a service contract (S1) and a fee-for-service practice context (S2); 139 participants were evaluated at $\mathrm{S} 1$ and 233 at S2, which began 2 years after the creation of the LI model at S1 (Tables 1 and 2). Participants at both sites were mainly women $(\sim 80 \%)$, with a mean age of 52 (standard deviation (SD) 13) years and 51 (SD 14) years for S1 and S2, respectively. Participants ranged in age from 16 to 85 years. Additional measures were available from participants at S1, including cardiometabolic indicators $(n=119)$ and mood scores $(n=111)$. More than $90 \%$ of participants had a high waist circumference, while the average baseline BMI was $37 \mathrm{~kg} /$ $\mathrm{m}^{2}$. Consistent with the high prevalence

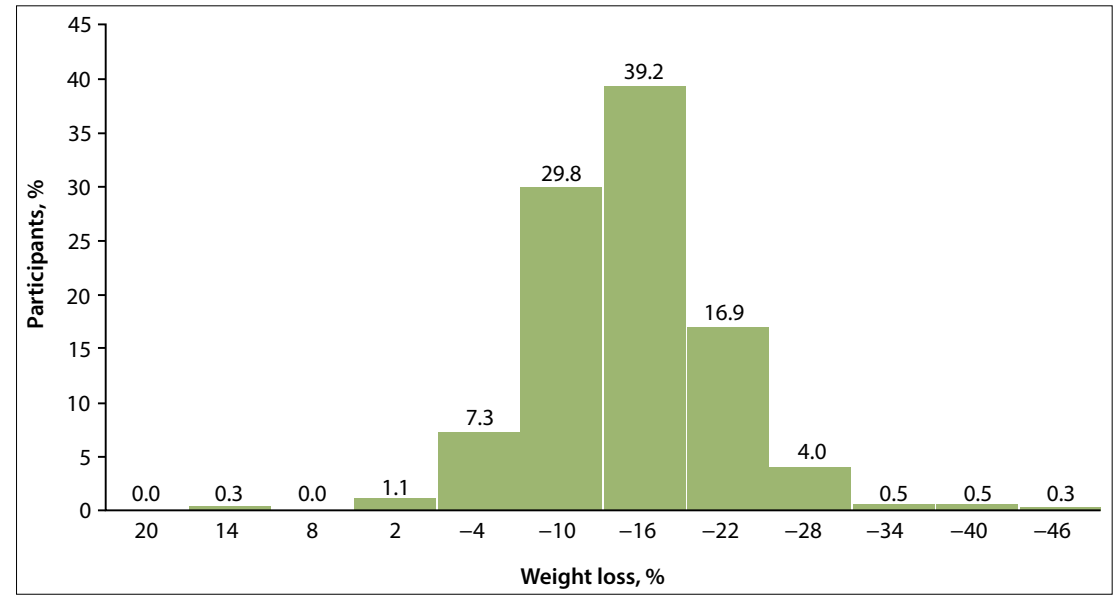

Fig. 1. Weight loss (\%) among participants in LIs in two primary care practices in rural British Columbia, Canada ( $\mathrm{N}=372)$.

Table 1. Characteristics at baseline and follow-up of participants at $\mathrm{S} 1$ in a service contract primary care practice in rural BC, Canada $(N=139)$

\begin{tabular}{lllll}
\hline Characteristic & Baseline & Follow-up & Change & $p$-value \\
\hline Age (years), mean (SD) & $52.4(13.1)$ & - & - & - \\
Sex, \% female & 80.4 & - & - & - \\
Height (m), mean (SD) & $1.7(0.1)$ & - & - & - \\
Weight (kg), mean (SD) & $97.2(22.6)$ & $84.2(20.6)$ & $-12.8(8.9)$ & $<0.0001$ \\
BMI (kg/m²), mean (SD) & $35.4(7.0)$ & $30.7(6.4)$ & $-4.7(3.2)$ & $<0.0001$ \\
\% with elevated waist circumference & 90.7 & 66.2 & -24.5 & $<0.0001$ \\
\% with metabolic syndrome & 57.6 & 19.4 & -38.2 & $<0.0001$ \\
\% with PHQ-9 score $\geq 10$ & 23.7 & 7.9 & -15.8 & $<0.0001$ \\
PHQ-9 score $(n=111)$, mean (SD) & $7.0(5.2)$ & $3.4(4.6)$ & $-3.6(4.6)$ & $<0.0001$ \\
Blood pressure (mmHg, $n=119)$, mean (SD) & $136.6 / 85.4$ & $122.5 / 77.0$ & $-14.1 / 8.4$ & $<0.0001$ \\
HDL-C (mmol/L, $n=119)$, mean (SD) & $1.34(0.35)$ & $1.42(0.35)$ & $0.08(0.27)$ & 0.0019 \\
LDL-C (mmol/L), mean (SD) & $3.31(1.04)$ & $2.90(0.88)$ & $-0.41(0.97)$ & $<0.0001$ \\
Triglyceride concentration (mmol/L), & $1.63(0.80)$ & $1.08(0.59)$ & $-0.56(0.64)$ & $<0.0001$ \\
mean (SD) & & & & \\
Triglyceride/HDL-C ratio, mean (SD) & $1.36(0.91)$ & $0.84(0.73)$ & $-0.52(0.77)$ & $<0.0001$ \\
Fasting blood glucose concentration & $5.91(1.74)$ & $5.32(1.17)$ & $-0.59(1.47)$ & $<0.0001$ \\
(mmol/L, $n=111)$, mean (SD) & & & & \\
HbAlc concentration (\%, $n=18)$, mean (SD) & $7.47(1.64)$ & $6.95(1.09)$ & $-0.52(1.91)$ & 0.089 \\
HDL-C = high-density lipoprotein cholesterol; LDL-C = low-density lipoprotein cholesterol. & & \\
& & & &
\end{tabular}

of obesity, $57.6 \%(80 / 139)$ of participants began the intervention at $\mathrm{S} 1$ with MetS while $12.9 \%(18 / 139)$ had T2DM.

Participants in these LIs had unusually large improvements in health, particularly given the real-world contexts of the interventions. For example, 372 participants had weight loss of $>12 \%$ (Fig. 1), while in the Diabetes Prevention Program, a wellresourced trial, the weight-loss goal was $7 \%$ of initial body weight. Among other studies examining LIs in routine practice, average weight loss was $3-5 \%$ at year $1 .^{[2]}$

Consistent with the considerable weight loss, participants at S1 showed marked improvements in their cardiometabolic profile. For example, blood triglyceride concentrations, measured among 119 participants at $\mathrm{S} 1$, decreased by $34 \%$, probably a reflection of the reduced intake of starches and sugars. ${ }^{[3]}$ Among the 18 individuals with $\mathrm{T} 2 \mathrm{DM}$ in the LI at S1, there was a mean decrease in $\mathrm{HbAlc}$ of $0.5 \%$, a figure that fails to account for any reductions in pharmacotherapy, which were not documented in this report. The extent of the changes in cardiometabolic indicators that were measured in this study are therefore a conservative estimate of the health improvements, as participants experienced reductions in the use of 
Table 2. Characteristics at baseline and follow-up of participants at S2 in a rural feefor-service primary care practice in $\mathrm{BC}$, Canada $(N=233)$

\begin{tabular}{lllll}
\hline Characteristic & Baseline & Follow-up & Change & $p$-value \\
\hline Age (years), mean (SD) & $51.3(14.1)$ & - & - & - \\
Sex, \% female & 81.6 & - & - & - \\
Height $(\mathrm{m})$, mean (SD) & $1.6(0.1)$ & - & - & - \\
Weight $(\mathrm{kg})$, mean (SD) & $101.0(21.4)$ & $89.5(19.4)$ & $-11.4(6.4)$ & $<0.0001$ \\
BMI $\left(\mathrm{kg} / \mathrm{m}^{2}\right)$, mean (SD) & $37.2(6.6)$ & $33.0(6.2)$ & $-4.3(2.5)$ & $<0.0001$ \\
$\%$ with elevated waist circumference & 98.3 & 83.3 & -15.0 & $<0.0001$
\end{tabular}

Table 3. Predictors of change in BMI among participants in an $\mathrm{LI}$ in rural $\mathrm{BC}$, Canada $(N=132)$

\begin{tabular}{lllll}
\hline Parameter & Change in BMI & Standard error & $p$-value & $\boldsymbol{R}^{\mathbf{2}}$ (full model = 0.23) \\
\hline Sex (women referent) & -0.236 & 0.646 & 0.7151 & 0.000 \\
$\begin{array}{l}\text { Age of participants } \\
\text { (years) }\end{array}$ & 0.036 & 0.019 & 0.0618 & 0.024 \\
Baseline BMI (kg) & 0.179 & & & \\
Months in programme & 0.138 & 0.035 & $<0.0001$ & 0.151 \\
No. of visits/month & 0.721 & 0.065 & 0.0347 & 0.018 \\
& & 0.254 & 0.0045 & 0.033
\end{tabular}

insulin and oral hypoglycaemic, antihypertensive and cholesterol-lowering agents. To the intervention participants, the reductions in pharmacotherapy were an empowering 'side-effect' of the intervention, and for the clinicians administering the intervention, use of this therapeutic approach improved control of hyperglycaemia, hypertension and dyslipidaemias.

Similar to the findings of others, ${ }^{[5,6]}$ we documented improvements in mood among participants in the intervention at $S 1(n=111)$. Among the 32 participants with mood scores indicative of depression (PHQ-9 score $\geq 10$ ), the mean decrease in score was 7.0 (SD 5.2). It was not possible to separate the effect of participating in group sessions from the physiological effects of the LI, as both these exposures are probably associated with improvements in mood. The mood improvements associated with weight loss may be attributable to reductions in pathophysiological processes such as inflammation and hypothalamic-pituitary-adrenal axis activation that are common to both insulin resistance and mood disorders. ${ }^{[7]}$

We examined the predictors of weight change among participants at S1 using multivariable analysis (Table 3). We found that each visit per month increase in the LI was associated with a $0.7 \mathrm{~kg} / \mathrm{m}^{2}$ greater loss in BMI after controlling for sex, age, baseline BMI and time spent in the programme. The importance of intensity of participation was reaffirmed in regression analysis by using change in weight per month as the dependent variable. These findings highlight the importance of group support in achieving therapeutic goals. Moreover, the clinical observation prior to the creation of the LI model was that one-on-one lifestyle counselling was less effective in producing lifestyle changes than participation in support groups. The effectiveness of support groups in the context of LIs has been documented previously ${ }^{[8]}$ and may indicate the contribution of food addictions to these conditions. ${ }^{[9]}$

\section{Conclusions}

We documented the creation of an LI model and the replication of this intervention in different rural practices. This intervention was a powerful wellness tool, empowering not only patients ${ }^{[10]}$ and physicians but the rural communities, which can be burdened with a high prevalence of chronic disease.

The intervention model documented in this study differed from the consensus prescription for LIs. For example, participants in this intervention were counselled to restrict moderate to vigorous physical activity while on the weight-loss diet; in contrast, in two highly cited randomised trials, participants were encouraged to undertake $150^{[1]}$ and $175^{[11]}$ minutes per week of moderate physical activity, respectively. More controversially, our intervention used a high-fat diet for weight maintenance, while in the Diabetes Prevention Program and the Look AHEAD trials, participants were counselled to avoid consuming foods rich in dietary fat. These conflicting prescriptions allude to a state of uncertainty that exists with regard to the optimal prescription for LIs for individuals with insulin resistance. This situation persists despite decades-old findings by Reaven (Garg et al. ${ }^{[12]}$ ) showing that insulin resistance is fundamentally a disorder of carbohydrate metabolism.

Given the magnitude of the obesity and diabetes pandemics, there is a public health imperative to provide practitioners with evidence that supports effective interventions. While well-resourced randomised trials are powerful analytical tools, rigorous trials take decades to yield results and are arguably prohibitively expensive. ${ }^{[13]}$ Moreover, study results often lack generalisability to routine practice. ${ }^{[14]}$ In contrast, the quality improvement process used in this study was not only powerful, as evidenced by the replication of the intervention model at four different practice sites, but offers a more expeditious way to spread effective interventions for obesity and insulin resistance.

Despite the rigour of our quality improvement process, our efforts to communicate the merits of this intervention to health system administrators met with a frustrating lack of uptake. This is not surprising, given that the research literature has many competing 'solutions' for the epidemics of obesity and diabetes, ${ }^{[15]}$ many of which are difficult to falsify. ${ }^{[16]}$ To support health administrators in making evidence-based decisions, a broader set of data sources could be used to evaluate health system interventions such as that documented here. For example, the Institute of Health Improvement recommends using indicators that measure patient satisfaction, health system cost and population health status, ${ }^{[17]}$ complementing data from physician records. A broader set of health system indicators combined with longer-term follow-up of intervention participants would enable evidence-based health system decision-making in a climate of fiscal restraint.

Taken as a whole, our evidence suggests that a timely response to the obesity and diabetes pandemics requires a critical rethink not only of the current evidence base underpinning LIs, but also of the systems with which evidence is generated and integrated into health system practice.

Conflicts of interest. SDT, KN, DC, MM SVDS and JF have no conflicts of interest to declare. SM is the founder of a sole proprietorship, Approach Analytics, providing analytical support to clinical and public health initiatives. JW is on the Scientific Advisory 
Board for Atkins Nutritionals Inc. and has accepted honoraria and travel expenses to attend meetings. TN is the author of the books Lore of Running and Waterlogged and co-author of The Real Meal Revolution, Raising Superheroes and Challenging Beliefs. All royalties from the sales of The Real Meal Revolution and Raising Superheroes and related activities are donated to the Noakes Foundation, of which he is the chairman and which funds research on insulin resistance, diabetes and nutrition as directed by its Board of Directors. Money from the sale of other books is donated to the Tim and Marilyn Noakes Sports Science Research Trust, which funds the salary of a senior researcher at the University of Cape Town, South Africa. The research focuses on the study of skeletal muscle in African mammals with some overlap to the study of type 2 diabetes in carnivorous mammals and of the effects of (scavenged) sugar consumption on freeliving (wild) baboons.

1. Orchard TJ, Temprosa M, Goldberg, R, et al. The effect of metformin and intensive lifestyle intervention on the metabolic syndrome: The Diabetes Prevention Program Randomized Trial. Ann Intern Med 2005;142(8):611-619. DOI:10.7326/0003-4819-142-8-200504190-00009

2. Kahn R, Davidson MB. The reality of type 2 diabetes prevention. Diabetes Care 2014;37(4):943-949. DOI:10.2337/dc13-1954

3. Shai I, Schwarzfuchs D, Henkin Y, et al. Weight loss with a low-carbohydrate, Mediterranean, or lowfat diet. N Engl J Med 2008;359(3):229-241. DOI:10.1056/NEJMoa0708681

4. Alberti KG, Eckel RH, Grundy SM, et al. Harmonizing the metabolic syndrome: A joint interim statement of the International Diabetes Federation Task Force on Epidemiology and Prevention
National Heart, Lung, and Blood Institute; American Heart Association; World Heart Federation; International Atherosclerosis Society; and International Association for the Study of Obesity. Circulation 2009:120(16):1640-1645. DOI:10.1161/circulationAHA.109.192644

5. McClernon FJ, Yancy WS, Eberstein JA, Atkins RC, Westman EC. The effects of a low-carbohydrate McClernon F, Yancy WS, Eberstein JA, Atkins RC, Westman EC. The effects of a low-carbohydrate
ketogenic diet and a low-fat diet on mood, hunger, and other self-reported symptoms. Obesity (Silver ketogenic diet and a low-fat diet on mood, hunger, and

6. Brinkworth GD, Noakes M, Buckley JD, Keogh JB, Clifton PM. Long-term effects of a very-lowcarbohydrate weight loss diet compared with an isocaloric low-fat diet after $12 \mathrm{mo}$. Am J Clin Nutr 2009;90(1):23-32. DOI:10.3945/ajcn.2008.27326

7. McIntyre RS, Soczynska JK, Konarski JZ, et al. Should depressive syndromes be reclassified as 'metabolic syndrome type II? Ann Clin Psychiatry 2007;19(4):257- 264. DOI:10.1080/10401230701653377

8. Jaber R, Braksmajer A, Trilling JS. Group visits: A qualitative review of current research. J Am Board Fam Med 2006;19(3):276-290. DOI:10.3122/jabfm.19.3.276

9. Avena NM, Rada P, Hoebel BG. Evidence for sugar addiction: Behavioral and neurochemical effects of intermittent, excessive sugar intake. Neurosci Biobehav Rev 2008;32(1):20-39. DOI:10.1016/j. neubiorev.2007.04.019

10. Lavoie C. Gestational diabetes: Poke, pee, and eat your carbs. Can Fam Physician 2011;57(7):756-757.

11. Look AHEAD Research Group, Wing RR, Bolin P, Brancati FL, et al. Cardiovascular effects of intensive lifestyle intervention in type 2 diabetes. N Engl J Med 2013;369(2):145-154. DOI:10.1056/NEJMoa1212914

12. Garg A, Bantle JP, Henry RR, et al. Effects of varying carbohydrate content of diet in patients with non-insulin-dependent diabetes mellitus. JAMA 1994;271(18):1421-1428. DOI:10.1001/
wark with non-insulin-dependen
jama.1994.03510420053034

13. Howard BV, van Horn L, Manson JE, et al. Low-fat dietary pattern and risk of cardiovascular disease: The Women's Health Initiative Randomized Controlled Dietary Modification Trial. JAMA 2006;295(6):655-666. DOI:10.1001/jama.295.6.655

14. Rothwell PM. External validity of randomised controlled trials: 'To whom do the results of this trial apply?' Lancet 2005;365(9453):82-93. DOI:10.1016/S0140-6736(04)17670-8

5. Taubes G. Prosperity's plague. Science 2009;325(5938):256-260. DOI:10.1126/science.325_256

6. Ioannidis JP. Why most published research findings are false. PLoS Med 2005;2(8):e124. DOI:10.1371/ journal.pmed.0020124

7. Berwick DM, Nolan TW, Whittington J. The triple aim: Care, health and cost. Health Aff 2008;27(3):759-769. DOI:10.1377/hlthaff.27.3.759

Accepted 6 June 2016 